\title{
NEXT GENERATION ADVANCED LIGHT SOURCE SCIENCE*
}

\author{
W.R.Flavell ${ }^{\#}$, School of Physics and Astronomy, The University of Manchester, Sackville St \\ Building, PO Box 88, Manchester M60 1QD, UK.
}

\begin{abstract}
Recent advances in accelerator science make feasible the provision of XUV and harder X-ray FELs that will generate short (fs regime) pulses of light that is broadly tuneable and $>10^{6}$ times more intense than spontaneous undulator radiation [1]. Energy recovery technology [2] offers the promise of short pulse, high peak flux spontaneous radiation, with particular advantages in the IR and $\mathrm{THz}$ parts of the spectrum. The new science enabled by these 4 th generation sources is reviewed. A key feature is dynamic measurements. Pump-probe experiments will allow real-time measurements of reaction pathways and short-lived intermediates. The high intensity of FEL radiation will allow very high resolution in imaging applications. The very high field intensity of the XUV radiation will lead to the creation of new states of matter, while at the highest X-ray energies, the goal is to achieve single molecule diffraction. Illustrations are provided of some of the experiments proposed in the Science Cases for the major world 4th generation projects. Some of the science already undertaken using IR and UV FELs, and results obtained from new XUV sources (such as FLASH at DESY [3]) are discussed.
\end{abstract}

\section{INTRODUCTION}

The enormous brightness and coherence of $4^{\text {th }}$ generation light sources means that we can only partially imagine what we might discover using these sources of ultra-short pulse light. These properties have led scientists around the world to consider the possibility that it might be possible to use them to control the direction of a chemical reaction, perhaps even to synthesise molecules (such as drugs) that are currently difficult or expensive to make. Already, workers at the Thomas Jefferson National Accelerator Facility, Virginia, have shown that their infrared (IR) FEL source is an ideal tool for synthesising high purity carbon nanorods - in economically viable kilogramme quantities - material that is difficult to make in a highly pure state by other routes [4]. The nanorods are being used by NASA in fibre-reinforced material for aerospace applications.

More generally, $4^{\text {th }}$ generation sources offer unique opportunities for dynamic experiments - enabling us to understand how molecules or devices function on timescales of tens of fs. This is the fundamental timescale on which molecules vibrate and on which bonds are made or broken, so many reaction pathways will be elucidated for the first time.

The high intensity and coherence of the sources also lead to powerful opportunities in imaging. In the infrared,

\footnotetext{
*Work supported by STFC (UK) \& NWDA (UK)

"wendy.flavell@manchester.ac.uk
}

the intensity is such that near-field imaging approaches may be adopted, allowing spectromicroscopy at resolutions below the diffraction limit, while in the hard $\mathrm{X}$-ray regime, the ultimate goal is single molecule diffraction, utilising the coherence of the source.

\section{PUMP-PROBE EXPERIMENTS}

A key question that has excited scientists is to what extent femtosecond FEL pulses may be used controllably to initiate a specific reaction and to follow its reaction pathway. At present, the mechanisms of very few reactions are understood in any detail - measurement techniques are usually too slow to monitor the intermediates formed. It's a bit like only being able to see who crosses the finishing line first in a race, and not how they got there. In order to be able to measure properly along a reaction trajectory, we need ultra-fast (femtosecond scale) pairs of synchronised light pulses one to start the reaction off, and another (at a fixed time delay) to measure what has happened (figure 1). By varying the time delay between the pump and the probe pulse, we can measure the reaction pathway directly.

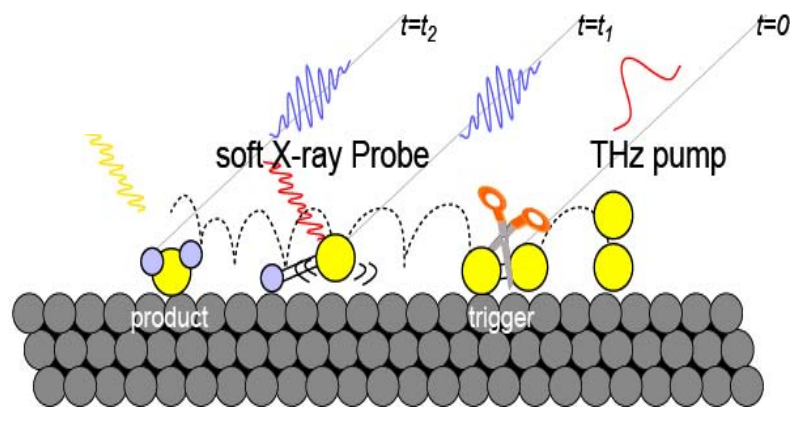

Figure 1: A pump-probe measurement at a surface. A very low energy terahertz $(\mathrm{THz})$ pulse causes initial dissociation of a molecule (yellow) at a surface at time $t_{0}$. The reaction is followed at times $t_{1}$ and $t_{2}$ using synchronised soft X-ray photons (blue) that monitor the reaction of the dissociated atoms with new species to form the product. (Adapted from original figure by Anders Nilsson and colleagues at SLAC [5]).

The generic term 'pump-probe' encompasses a vast range of dynamic experiments. The pump pulse may range in frequency from $\mathrm{THz}$, with energies of the order kT (for example in initating a room temperature surface reaction as shown in figure 1 [5], or in promoting proton transfer in enzyme catalysis [6]) to those in the VUV or XUV (for example in investigating hydrocarbon combustion [7] or reactions in the interstellar medium [6]). The probe pulse may range in frequency from $\mathrm{THz}$ (for example in measuring the time evolution of the excitonic states of a semiconductor [8]) to soft X-ray 
(giving chemical specificity in probing molecular intermediates, as in figure 1) or hard X-rays, giving timeresolved structural information [9].) A key advantage of $4^{\text {th }}$ generation sources over table-top sources in this application is that they offer laser-like radiation in important frequency regimes that are not well-covered by table-top lasers, in particular in the VUV, XUV and ultimately hard X-ray regimes, and in the far-IR and $\mathrm{THz}$ parts of the spectrum.

\section{Pump-probe experiments at FLASH}

Currently the only short wavelength FEL in operation in the world is the FLASH facility at the DESY laboratory near Hamburg, operating in the range 6.5-60 nm. It will remain so until 2009 when LCLS in Stanford, USA, and FERMI@ELETTRA are expected to start operation. It is already being used for important tests of theory - for example, in the very first results published in 2002 , clusters of Xe atoms were shown to undergo a Coulomb explosion in the huge field provided by the FEL pulses at much lower fluence than is predicted by available theory [3]. More recently, Michael Meyer and his co-workers have been able to carry out pump-probe experiments of the ionisation of He using XUV photons from FLASH synchronised with a ps optical table-top laser (Figure 2) [10]. When the two pulses overlap, new 'sideband' peaks appear in the photoemission spectrum, due to the dressing of the continuum electrons with one or more optical photons. This effect may be used as a pulse diagnostic to identify the time delay between the pump and probe pulses as the intensity of the side bands varies with the degree of temporal overlap between the XUV and dressing pulses [11].

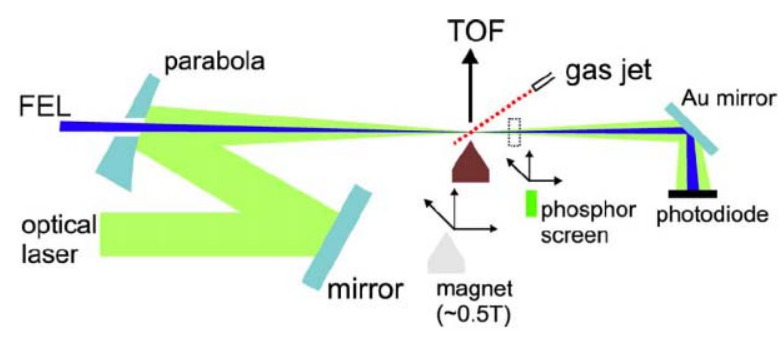

Figure 2: Schematic experimental arrangement for a gasphase pump-probe experiment using the FLASH facility at the DESY Laboratory in Hamburg in combination with an optical laser. 'One-shot' experiments are possible using time-of-flight (TOF) detection of the electrons produced. Adapted from reference [10] and reproduced with permission of M Meyer.

In more recent experiments, these workers use this approach in two-colour above-threshold ionisation of $\mathrm{He}$ and $\mathrm{Xe}$ to determine the jitter between the two independent photon sources; 20 fs XUV FEL pulses from FLASH and a 120 fs $800 \mathrm{~nm}$ Ti:sapphire laser. A $250 \mathrm{fs}$ rms jitter is found [12].

\section{Towards quantum chemical control; progress using table-top lasers}

The progress made already at FLASH is occurring at a time when rapid advances are being made in pulseshaping using adaptive control [13] and molecular alignment using table-top lasers [14]. Field-free molecular alignment of $\mathrm{SO}_{2}$ molecules has recently been demonstrated using two time-separated fs laser pulses of perpendicular polarisation at $815 \mathrm{~nm}$ - the first produces $1 \mathrm{D}$ alignment of the most polarisable axis of the molecule, at which point the second produces a torque about this axis that brings the seond most polarisable axis of the molecule into alignment with the field of the second laser pulse [14]. Using pairs of fs pulses whose relative phase is locked on attosecond timescales, together with another fs probe pulse, Kiyoshi Ueda and co-workers have observed the quantum interference of two molecular wavepackets in real-time in an excited state of the $\mathrm{I}_{2}$ molecule [15]. The evolution of this interference in real time is shown to be dependent on the relative phases of the locked pulses [15].

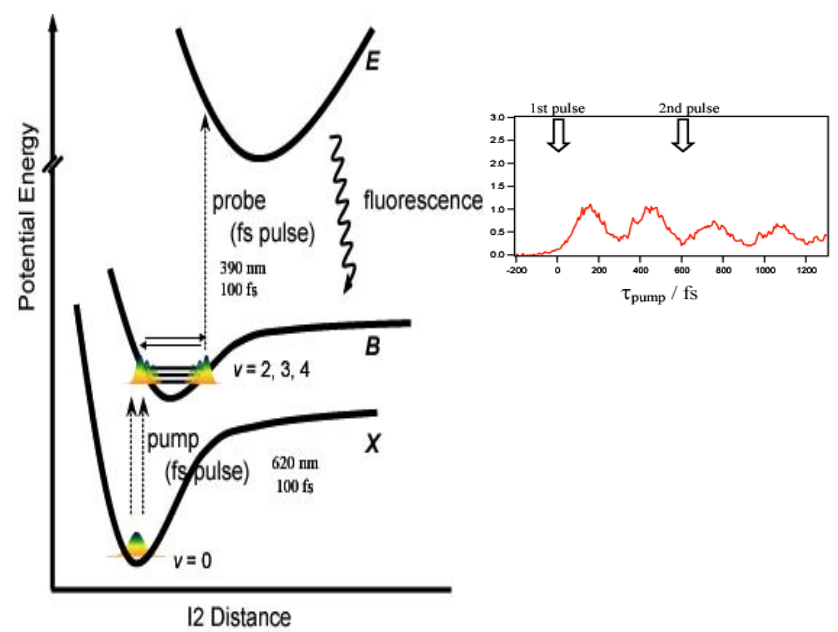

Figure 3: Pump-control-probe scheme for real-time measurement of wavepacket interference using fs pulses in the B-state of the $\mathrm{I}_{2}$ molecule [15]. The quantum interference is detected as oscillations in the fluorescence signal as a function of the time delay between the pump and probe pulses, $\tau_{\text {probe }}$ (inset, right). Reproduced with permission of Kiyoshi Ueda.

Experiments such as these begin to allow access to the phase and amplitude information contained in a wavepacket, and open up possibilities for coherent control of a variety of quantum systems - with the long term goal of using shaped light pulses to control the direction of a chemical reaction. These experiments are possible using table-top lasers because of the attosecond control of the relative phase of an IR fs pulse that may be achieved using nonlinear optical materials. A lack of suitable materials above around $6 \mathrm{eV}$ remains a challenge to achieving the necessary pulse-shaping for quantum chemical control with VUV and XUV FEL sources. 
Possible approaches such as the use of phase-coherent double-pulse sequences using a VUV interferometer have been proposed [16].

\section{Reaction initiation at room temperature}

Many of the important reactions that we would like to understand occur at very close to room temperature - an example is proton transfer in enzyme catalysis - and the question that then arises is can we really use a low energy IR FEL pulse to promote a specific reaction? Surely all the energy in the pulse will immediately be dissipated by internal vibrational relaxation (IVR), and we will just see a non-specific warming effect? One of the first indications that this is not the case came in 2006 from experiments by Norman Tolk and his co-workers using the Vanderbilt FEL in the US [17].
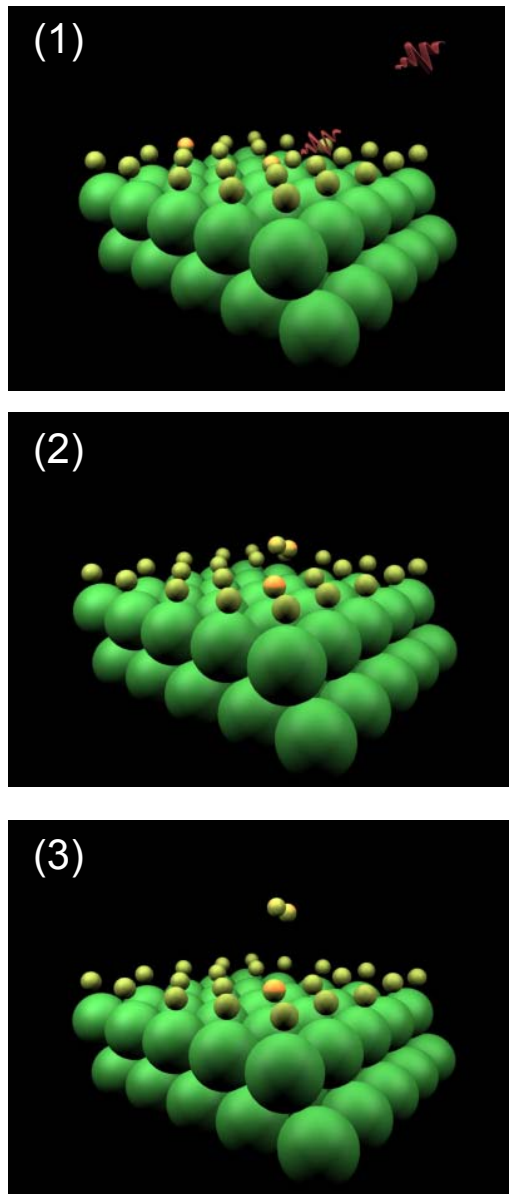

Figure 4: FEL-induced hydrogen desorption from a Si (111) surface. Si atoms are shown in green, and $\mathrm{H}$ in yellow. FEL photons come in and one photon excites one hydrogen adatom (1). Two excited neighbouring hydrogen adatoms pair up and as a result of "energy pooling" desorb associatively at room temperature (2) \& (3). Figure reproduced with permission of N H Tolk [17].

They took a silicon (111) surface covered in hydrogen atoms, and gradually tuned the IR FEL to the frequency of the Si-H stretching vibration at around $4.8 \mu \mathrm{m}$. They saw a large rise in the desorption of hydrogen molecules as they hit this threshold. When they substituted deuterium for some of the hydrogen and measured the amounts of $\mathrm{H}_{2}, \mathrm{D}_{2}$ and $\mathrm{HD}$ emerging they discovered that the proportions were very far from what they would expect if the IR FEL energy was being dissipated throughout the system, and the reaction was occurring via the normal 'thermal' pathway. Their conclusion is that desorption of hydrogen only occurs when two adjacent $\mathrm{Si}-\mathrm{H}$ bonds are simultaneously excited, and the two $\mathrm{H}$ atoms associate and are desorbed through 'energy pooling' (figure 3) [17]. These experiments are exciting as they suggest that site-selective chemistry can be carried out at room temperature - perhaps leading to new methods of hydrogen storage or to room temperature refining of hydrocarbons.

Studies such as these lend credence to the idea of using $\mathrm{THz}$ or IR pulses to initiate or promote specific dynamic processes. An example where such studies may have immediate applicability is in experimental studies of quantum-mechanical tunnelling in proton transfers in enzyme reactions. This is one of a number of areas in molecular biology where ab initio calculation is ahead of experiment. Molecular dynamics simulations of quinoproteins suggest that the fast proton transfers observed in oxidative deamination occur through proton transfers mediated by a 'promoting vibration' [18] that acts to narrow the activation barrier to reaction and thus enhance tunnelling. The nature of the promoting vibration (whether short or long-range) is controversial. The relevant frequencies lie in the far-IR/THz, so the highly intense broadband $\mathrm{THz}$ radiation provided by energy recovery linac (ERL) sources such as that at JLab, US [2], or now coming onstream at Daresbury, UK (ERLP/4GLS [6]) may provide the ideal source for a new generation of non-damaging macromolecular dynamics experiments.

\section{NEAR-FIELD APPROACHES TO BREAKING THE DIFFRACTION LIMIT}

The high intensity of FEL sources allows for imaging modalities to be implemented that use near-field techniques to image at resolutions below the diffraction limit. This is particularly important in the infrared where spectromicroscopy may allow for simultaneous imaging with chemical mapping of the species present, but where the long wavelength of the radiation would otherwise limit the spatial resolution to $\mu \mathrm{m}$ lengthscales. This low frequency radiation is non-ionising, and so in principle offers the opportunity for imaging and spectromicroscopy of live tissue. A particularly important example is IR spectromicroscopy of cells at sub-cellular resolution [19]. Figure 5 shows an example of the work of Antonio Cricenti and co-workers, obtained using scanning near-field optical microscopy (SNOM) at the Vanderbilt IR FEL facility.

The SNOM technique may be subject to artefacts created by the sample topology and inhomogeneities. A 
number of alternative approaches have been implemented in recent years to try to overcome this problem; an example is the 'AFMIR' acousto-optical technique, where an atomic force microscope tip probes the transient local deformation produced by IR FEL pulses tuned to one of the sample absorptions [20]. The spatial resolution has been shown to be better than $\lambda / 100$.

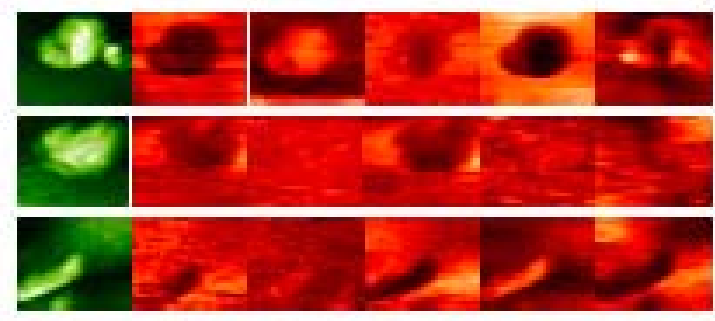

Figure 5: Scanning near-field techniques used with the Vanderbilt University IR-FEL source to analyze the distribution of functional groups in a single cell with a resolution well below the diffraction limit. The lefthand column shows topography images for $\mathrm{HaCat}$ (top), pancreatic (middle) and Cos 7 (bottom) cells, with the corresponding IR images at frequencies from $6.1 \mu \mathrm{m}$ (amide $\mathrm{C}=\mathrm{O}$ stretch) to $8.05 \mu \mathrm{m}$ (phosphorus stretch) shown to the right. (Antonio Cricenti, ISM-CNR, RomaItaly and Vanderbilt University, Nashville, USA, reproduced with permission.)

\section{TIME-RESOLVED AND SINGLE MOLECULE DIFFRACTION}

The imminent advent of hard X-ray FELs, such as LCLS and XFEL offers the opportunity to record diffraction information in a time-resolved way - for example to study the fundamentals of surface or cluster melting on fs timescales. A long-term goal of the hard Xray FEL projects is to use the coherence of the source to achieve the goal of single molecule diffraction - in particular to be able to determine the structure of a single biomolecule (e.g. a large macromolecule or virus) in a molecular jet, without the constraint of having to produce a single crystal sample. These experiments will be technically very demanding, requiring some years of development after the hard X-ray FELs become available, but the information they yield will be enormously valuable in biology, where the majority of proteins (for example) cannot be crystallised. The high energy and high fluence of the pulses from the X-ray FELs will result in the molecule or cluster under study being destroyed by undergoing a 'Coulomb explosion' and turning into a plasma on timescales of tens of fs $[3,21,22]$. The experiment is thus intrinsically a 'one-shot' experiment, and data must be gathered from an individual molecule before it is destroyed, requiring fast detection and data readout. In the absence of the crystalline order of the lattice giving systematic absences, the data are 'oversampled' and it is possible to use techniques developed from electron diffraction to retrieve the phase information necessary to allow image reconstruction from the coherent pattern [23]. In the case of a large macromolecule, virus or cell having dimensions up to the $\mu \mathrm{m}$ range, it may be possible to extract a single diffraction pattern from one pulse. However, calculations show that even the intensity from an XFEL will not be enough to allow the image to be reconstructed from one pulse in the case of a smaller biomolecule [24]. It should nevertheless be enough to allow the orientation of the molecule to be determined. The experiment would then be repeated on a series of molecules (perhaps some hundreds), and the resulting images oriented and added to achieve sufficient signal-to-noise level. This would be simplified if the molecules could be pre-oriented in a molecular jet [24].

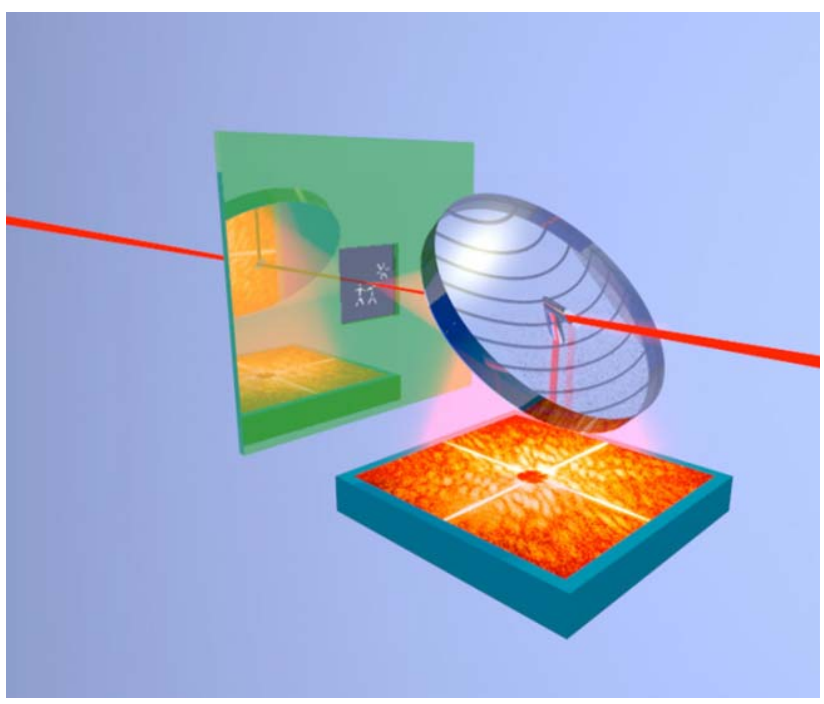

Figure 6: Schematic diagram of the FLASH 'single-shot' diffraction experiment. A FEL beam incident from left to right passes through a $20 \mathrm{~nm} \mathrm{SiN} \mathrm{membrane} \mathrm{with} \mathrm{a} \mu \mathrm{m}$ scale image milled through its thickness. The direct beam passes through the sample window and exits the camera through a hole in a graded multilayer planar mirror. The diffracted light from the sample reflects from this mirror onto a CCD camera. Under the conditions of the experiment, the on axis pathlength from the sample to the detector is in the far field, where the diffraction pattern is equal to the Fourier transform of the exit wave. Image source DESY, Hamburg, H N Chapman, J Hajdu et al.

Given the technical challenges involved in delivering this experiment, it is important that development work begins as soon as possible, and the proposers of this experiment have already made a promising start in using the FLASH facility for imaging larger structures with a dimension commensurate with the wavelengths (tens of $\mathrm{nm}$ ) delivered by the source [25]. They have used $25 \mathrm{fs}$, $32 \mathrm{~nm}$ pulses delivering $4 \times 10^{13} \mathrm{~W} \mathrm{~cm}^{-12}\left(10^{12}\right.$ photons per pulse) to produce a coherent image from a nonperiodic micron-sized pattern cut into a $\mathrm{SiN}$ membrane before it was destroyed. The experimental arrangement is shown in figure 6 . The reconstructed image is obtained at diffraction-limited resolution and shows no evidence of damage [25]. 


\section{CONCLUSIONS}

Next generation light sources will offer unparalleled insights into the dynamic behaviour of matter at the atomic level. The advantages in intensity, coherence and pulse length over present accelerator sources mean that we can only dimly grasp the full potential at the outset of this revolution. Particularly in the XUV, where the field intensity is sufficient to allow the creation of new states of matter [24], we will enter uncharted territory.

\section{ACKNOWLEDGEMENTS}

Thanks are due to Norman Tolk, Michael Meyer, Antonio Cricenti, Kiyoshi Ueda and Anders Nilsson for allowing reproduction of their figures. Financial support from STFC (UK) is gratefully acknowledged.

\section{REFERENCES}

[1] e.g. J Andruszkow et al., Phys. Rev. Lett., 85, 3825, (2000).

[2] e.g. G.L Carr, M C Materin, W R McKinney, K Jordan, G R Neil and G P Williams, Nature, 420, 153 (2002).

[3] e.g. H Wabnitz, L Bittner, A R B de Castro, R Döhrmann, P Gürtler, T Laarmann, W Laasch, J Schulz, A Swiderski, K von Haeften, T Möller, B Faatz, A Fateev, J Feldhaus, C Gerth, U Hahn, E Saldin, E Schneidmiller, K Sytchev, K Tiedtke, R Treusch and M Yurkov, Nature, 420, 482, (2002).

[4] http://www.jlab.org/FEL/carbonnano.html.

[5] Anders Nilsson, presentation at the 4GLS Users' Consultation Meeting, $4^{\text {th }}$ July 2005 , available at www.4gls.ac.uk.

[6] 4GLS Conceptual Design Report, April 2006, available at www.4gls.ac.uk.

[7] C A Taatjes, N Hansen, A Mcllroy, J A Miller, J P Senosiain, S J Klippenstein, F Qi, L Sheng, Y Zhang, T A Cool, J Wang, P R Westmoreland, M E Law, T Kasper and K Kohse-Höinghaus, Science, 308, 1187, (2005).

[8] e.g. R A Kaindl, M A Carnahan, D Hägele, R Lövenich and R S Chemla, Nature, 423, 734 (2003).

[9] e.g. W Gawelda, V-T Pham, M Benfatto, Y Zaushitsyn, M Kaiser, D Grolimund, S L Johnson, R Abela, A Hauser, C Bressler and M Chergui, Phys. Rev. Lett., 98, 057401 (2007).

[10] M Meyer, D Cubaynes, P O'Keeffe, H Luna, P Yeates, E T Kennedy, J T Costello, P Orr,R Taïeb, A Maquet, S Düsterer, P Radcliffe, H Redlin, A Azima, E Plönjes and J Feldhaus, Physical Review A, 74, 011401(R), (2006).

[11] S Düsterer, P Radcliffe, G Geloni, U Jastrow, M Kuhlmann, E Plönjes, K Tiedtke, R Treusch, J Feldhaus, P Nicolosi, L Poletto, P Yeates, H Luna, J
P Costello, P Orr, D Cubaynes and M Meyer, Opt. Lett., 31, 1750, (2006).

[12]P Radcliffe, S Düsterer, A Azima, H Redlin, J Feldhaus, J Dardis, K Kavanagh, H Luna, J Pedregosa Gutierrez, P Yeates, E T Kennedy, J T Costello, A Delserieys, C L S Lewis, R Taïeb, A Maquet, D Cuaynes and M Meyer, Appl. Phys. Lett., 90, 131108, (2007).

[13]C Daniel, J Full, L Gonzalez, C Lupulescu, J Manz, A Merli, S Vajda and L Wöste, Science 299536 (2003).

[14] K F Lee, D M Villeneuve, P B Corkum, A Stolow and J G Underwood, Phys. Rev. Lett., 97, 173001 (2006).

[15] Kenji Ohmori, Hiroyuki Katsuki, Hisahsi Chiba, Masahiro Fujiwara, Yukinori Sato and Kiyoshi Ueda, Phys. Rev. Lett., 96, 093002 (2006).

[16]Kiyoshi Ueda, contribution to the 4GLS Science Case.

[17]Zhiheng Liu, L C Feldman, N H Tolk, Zhenyu Zhang, P I Cohen, Science, 312, 1024, (2006).

[18]L Masgrau, A Roujeinikova, L O Johannissen, P Hothi, J Basran, K E Ranaghan, A J Mulholland, M J Sutcliffe, N S Scrutton and D Leys, Science, 312, 237, (2006).

[19] A Cricenti, R Generosi, M Luce, P Perfetti, G Margaritondo, D Talley, J S Sanghera, I D Aggarwal, N H Tolk, A Congiu-Castellano, M A Rizzo and D W Piston, Biophysical J., 85, 2705 (2003).

[20] A Dazzi, R Prazeres, F Glotin and J M Ortega, Optics Letters, 30, 2388 (2005).

[21] R Neutze, R Wouts, D van der Spoel, E Weckert and J Hadju, Nature, 406, 752 (2000).

[22] T Laarmann, M Rusek, H Wabnitz, J Schulz, A R B de Castro, P Gürtler, W Laasch and T Möller, Phys. Rev. Lett., 95, 063402 (2005).

[23] J Miao, P Charalambous, J Kirz and D Sayre, Nature, 400, 342 (1999).

[24] TESLA Technical Design Report, Part V The X-ray Free Electron Laser, eds G. Materlik, Th. Tschentscher, March 2001, XFEL Technical Design Report, $2^{\text {nd }}$ draft, Eds Massimo Altarelli et al., May 2006.

[25] H N Chapman, A Barty, M J Bogan, S Boutet, M Frank, S P Hall-Riege, S Marchesini, B W Woods, S Bajit, W H Benner, R A London, E Plönjes, M Kuhlmann, R Treusch, S Düsterer, T Tschentscher, J R Schneider, E Spiller, T Möller, C Bostedt, M Hoener, D A Shapiro, K O Hodgson, D van der Spoel, F Burmeister, M Bergh, C Claeman, G Huldt, M M Seibert, F R N C Maia, R W Lee, A Szöke, N Timneanu and J Hajdu, Nature Phys., 2, 839, (2006). 